\title{
ON CALCULATION OF THE RELATIVE INDEX OF A FIXED POINT IN THE NONDEGENERATE CASE
}

\author{
A. V. GUMINSKAYA AND P. P. ZABREIKO
}

Received 26 June 2005; Accepted 1 July 2005

The paper is devoted to the calculation of the index of a zero and the asymptotic index of a linear completely continuous nonnegative operator. Also the case of a nonlinear completely continuous operator $A$ whose domain and image are situated in a closed convex set $Q$ of a Banach space is considered. For this case, we formulate the rules for calculating the index of an arbitrary fixed point and the asymptotic index under the assumption that the corresponding linearizations exist and the operators of derivative do not have eigenvectors with eigenvalue 1 in some wedges.

Copyright (C 2006 A. V. Guminskaya and P. P. Zabreiko. This is an open access article distributed under the Creative Commons Attribution License, which permits unrestricted use, distribution, and reproduction in any medium, provided the original work is properly cited.

\section{Introduction}

Let $Q$ be a closed convex set in a Banach space and let $A: Q \rightarrow Q$ be a completely continuous operator. In [4] the calculation problem of a fixed point index of a vector field $I-A$ was formulated. In the simplest case, when $Q$ is a cone, this problem was investigated in the articles by Isaenko [3], Mukhamadiev and Sabirov [5], and Pokornyi [6] (see also references in $[8]$ ). Later, in $[1,2]$ Dancer presented the general formula for the fixed point index of a completely continuous operator $A$ with its domain and image in an arbitrary closed convex set. However, the case of an asymptotic index was not considered. Note that for the case of a cone this problem was earlier considered in the articles by Pokornyi [6] and Pokornyi and Astaf'eva [7]. The present paper concerns the cases of a wedge and an arbitrary closed convex set. In the latter case, the calculation of a fixed point index is reduced to the index calculation with respect to a specially constructed wedge. We also show that for the infinity singular point one needs to take the wedge

$$
W_{\infty}=\{h \in X: x+t h \in Q(x \in Q, 0 \leq t<\infty)\} .
$$

Hindawi Publishing Corporation

Abstract and Applied Analysis

Volume 2006, Article ID 86173, Pages 1-11

DOI 10.1155/AAA/2006/86173 


\section{Index of a linear operator}

Let $X$ be a Banach space, $W$ a wedge in $X$ (this means that $W$ is a closed subset of $X$ such that $W+W \subseteq W$ and $\lambda W \subset W$ for $\lambda \geq 0$ ), and $A$ a linear completely continuous operator such that

$$
A W \subseteq W
$$

Let $L=W \cap(-W)$. Then $L$ is the maximal subspace which is contained in $W$. From (2.1) and the linearity of $A$ it follows that the inclusion

$$
A(L) \subseteq L
$$

holds.

Consider a quotient space $X / L$ and a quotient mapping $[\cdot]: X \rightarrow X / L$. It is easy to check that the image $\widehat{W}$ of $W$ under the quotient mapping [ [ ] is a cone in $X / L$.

From (2.2) it follows that the operator $A$ induces a linear mapping $\hat{A}$ of the quotient space $X / L$ into itself such that $\hat{A}[x]=[A x]$. And by $(2.1)$ we have that the cone $\widehat{W}$ is invariant under the operator $\hat{A}$, that is, $\hat{A}$ is nonnegative in the quotient space $X / L$.

Theorem 2.1. Let $A$ be a linear completely continuous operator, acting in a Banach space $X$, and let $W$ be a wedge that is invariant under the operator A. Suppose that $A x \neq x$ for $x \in W, x \neq 0$. Then $\rho(\hat{A}) \neq 1$ and

$$
\operatorname{ind}(0, I-A ; W)=\operatorname{ind}(\infty, I-A ; W)= \begin{cases}(-1)^{\beta\left(\left.A\right|_{L}\right)} & \text { if } \rho(\hat{A})<1, \\ 0 & \text { if } \rho(\hat{A})>1,\end{cases}
$$

where $\left.A\right|_{L}$ is the restriction of the operator $A$ to the space $L, \beta\left(\left.A\right|_{L}\right)$ is the sum of multiplicities of eigenvalues of $\left.A\right|_{L}$, greater than 1 , and $\rho(\hat{A})$ is the positive spectral radius of the operator $\widehat{A}$.

Proof. If $A x \neq x$ for $x \in W, x \neq 0$, then zero and infinity singular points of the vector field $\Phi=I-A$ are isolated in $W$. Hence the relative indices ind $(0, I-A ; W)$ and ind $(\infty, I-$ $A ; W)$ are well posed. In this case, by the definition of index at infinity (see, e.g., [4]), since the operator $A$ has no more fixed points in $W$, it follows that ind $(\infty, I-A ; W)=$ ind $(0, I-A ; W)$.

To calculate the index ind $(0, \Phi ; W)$ we will consider two possible cases: when the spectral radius $\rho(\hat{A})$ of the operator $\widehat{A}$ is less than 1 and when it is greater than 1 . The case $\rho(\widehat{A})=1$ is impossible. Indeed, if this is not true, then there exists an element $[x]^{*} \neq 0$ of the quotient space $X / L$ such that $\hat{A}[x]^{*}=[x]^{*}$. In other words, there exist $u \in W, u \notin L$, and $y \in L$ such that the equality $u-A u=y$ holds. Let us explore the solvability in $L$ of $(I-A) w=y$. If it had a solution $w \in L$, then the vector $u-w$ would be an eigenvector of the operator $A$, corresponding to the eigenvalue 1 and would be in $W$, which would contradict our assumptions. If we supposed that the equation had no solutions in $L$, then the operator $I-A$ would be invertible in $L$, which is impossible.

In the case $\rho(\hat{A})<1$, let us show that the vector field $\Phi x=x-A x$ is linearly relatively homotopic on $S_{W}=\{x \in W:\|x\|=1\}$ to the field $\Phi_{1} x=x-A Q(x)$, where $Q: X \rightarrow L$ is 
a projection (in general, nonlinear) of $X$ on $L$ (see [4, Theorem 18.1]). To prove this fact assume the converse, that is, that

$$
x=(1-\lambda) A x+\lambda A Q x
$$

has a solution for some $x \in S_{W}$ and $\lambda \in[0,1]$. Now if we rewrite this equation for the operator $\widehat{A}$ and recall (2.2), we get

$$
[x]=(1-\lambda) \hat{A}[x] \quad\left([x] \in \widehat{S}_{W}, \lambda \in[0,1]\right),
$$

where $\widehat{S}_{W}=\{[x] \in \widehat{W}:\|[x]\|=1\}$.

Under our assumption on the spectral radius of the operator $\hat{A}$ this yields $[x]=0$. In this case, we obtain $x=Q x$ and then (2.4) implies $x=A x$ for $x \in S_{W}$, which contradicts the assumption of our theorem.

Thus the relative index ind $(0, I-A ; W)$ is equal to the relative index ind $(0, I-A Q ; W)$, which actually is the Leray-Schauder zero fixed point index of the restriction $\left.A\right|_{L}$ of the operator $A$ to the subspace $L$ and we can calculate it by the well-known formula (see, e.g., [4, Theorem 21.1])

$$
\operatorname{ind}(0, I-A Q ; W)=(-1)^{\beta\left(\left.A\right|_{L}\right)},
$$

where $\beta\left(\left.A\right|_{L}\right)$ is the sum of multiplicities of eigenvalues of $\left.A\right|_{L}$, greater than 1 .

Now assume that $\rho(\widehat{A})>1$. In this case, there exists an element $[x]^{*}$ in the cone $\widehat{W}$ such that $\hat{A}[x]^{*}=\rho[x]^{*}(\rho>1)$. In other words, there exist an element $x^{*} \notin L$ of the wedge $W$ (we assume that $\left\|x^{*}\right\|=1$ ) and an element $z$ of the subspace $L$ such that $A x^{*}=\rho x^{*}+z$.

Show that the vector field $\Phi x=x-A x$ is linearly relatively homotopic on $S_{W}$ to the field $\Phi_{2} x=x-c x^{*}$, where the constant $c$ will be defined later. Let us show that

$$
x=(1-\lambda) A x+\lambda c x^{*}
$$

has no solutions for $x \in S_{W}, \lambda \in[0,1]$.

For $\lambda=0,(2.7)$ coincides with $x=A x$. The latter equation has no solutions for $x \in S_{W}$. For $0<\lambda \leq 1$ from (2.7), it follows that there exists a real $t>0$ such that

$$
x \geq t x^{*}
$$

We claim that there exists the maximal of such reals: $\xi=\max _{x \in S_{W}, x \geq t x^{*}} t$. We argue by contradiction. The inequality $x^{*} \leq x / t$ implies that if $t$ tends to infinity, then $x^{*} \leq 0$. On the other hand, $x^{*}$ is an element of the wedge $W$, thus $x^{*} \geq 0$. Hence we get that $x^{*} \in L$, which contradicts its choice.

Further, (2.7) and (2.8) imply

$$
x \geq(1-\lambda) \xi \rho x^{*}+\lambda c x^{*}=((1-\lambda) \xi \rho+\lambda c) x^{*} .
$$

Choose $c>\xi$ and observe that for this $c$ the inequality $(1-\lambda) \xi \rho+\lambda c>\xi$ holds. Indeed, for $\lambda=0$ this follows from $\rho>1$. For $\lambda=1$ this follows from our assumption for $c$. For other $\lambda \in(0,1)$ the inequality holds as the result of two previous cases. 


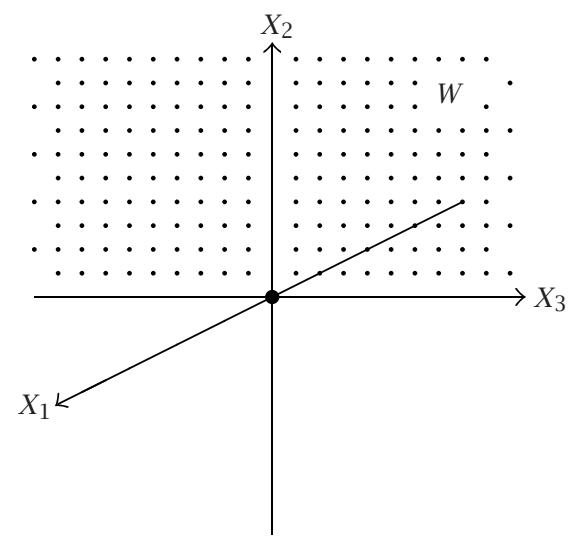

Figure 2.1

Therefore for the chosen $c$, the real $\xi$ is not maximal among $t$ such that $x \geq t x^{*}$. This contradiction proves the nondegeneracy of the linear homotopy connecting vector fields $\Phi$ and $\Phi_{2}$. Consequently, ind $(0, \Phi ; W)=\operatorname{ind}\left(0, \Phi_{2} ; W\right)$.

To calculate ind $\left(0, \Phi_{2} ; W\right)$ we will use the corollary of the Hahn-Banach theorem. According to it there exists a functional $l \in X^{*}$ such that $l(W) \geq 0$ and $l\left(x^{*}\right)=1$ (then $\|l\| \geq 1$ ). Since (2.8), we obtain $\xi \leq l(x) \leq\|l\|$ for $x \in S_{W}$. And our assumption of $c$ yields $c>\|l\| \geq 1$. Now we can show that the vector field $\Phi_{2} x=x-c x^{*}$ is nondegnerate on $\mathrm{cl} B_{W}=\{x \in W:\|x\| \leq 1\}$. Indeed,

$$
\left\|x-c x^{*}\right\| \geq\left\|c x^{*}\right\|-1=c-1>0
$$

Hence, by the relative rotation property, ind $\left(0, \Phi_{2} ; W\right)=0$. This completes the proof.

In applications there usually exist a complement $X_{1}$ of the linear hull $L(W)=W-W$ of the wedge $W$ to $X$ and a complement $X_{2}$ of a maximal subspace $X_{3}=W \cap(-W)$ in $W$ to $L(W)$. Then $X$ can be presented as the direct sum of subspaces (see Figure 2.1)

$$
X=X_{1} \dot{+} X_{2} \dot{+} X_{3} \text {. }
$$

From (2.1) and the linearity of the operator $A$ it follows that the inclusions

$$
A\left(X_{2} \dot{+} X_{3}\right) \subseteq X_{2} \dot{+} X_{3}, \quad A\left(X_{3}\right) \subseteq X_{3}
$$

hold.

Assume that an intersection $W \cap X_{2}$ is not empty. Then it is easy to prove that this set is a cone $K$ in $X_{2}$. It generates the order relation in $X_{2}$ by the following rule: $x \leq y$ if $y-x \in K$. The cone $K$ can be set as $K=\left\{x \in X_{2}: x \geq 0\right\}$. 
It can be proved that under such decomposition of the space $X$, the wedge $W$ is invariant under a linear operator $A$ if and only if $A$ is determined by the matrix

$$
A=\left(\begin{array}{ccc}
a_{11} & 0 & 0 \\
a_{21} & a_{22} & 0 \\
a_{31} & a_{32} & a_{33}
\end{array}\right)
$$

where $a_{22} \geq 0$. Furthermore, one can show that zero and infinity singular points of such operator $A$ are isolated in $W$ if and only if 1 is not an eigenvalue of the operators $a_{22}$ and $a_{33}$. In this case, Theorem 2.1 can be formulated in the following way.

Theorem 2.2. Let $A$ be a linear completely continuous operator, acting in a Banach space $X$, and let $W$ be a wedge that is invariant under the operator $A$. Then $A$ can be defined by matrix (2.13). If 1 is not an eigenvalue of the operators $a_{22}$ and $a_{33}$, then zero and infinity singular points of the operator $A$ are isolated in $W$ and

$$
\operatorname{ind}(0, I-A ; W)=\operatorname{ind}(\infty, I-A ; W)= \begin{cases}(-1)^{\beta\left(a_{33}\right)} & \text { if } \rho\left(a_{22}\right)<1 \\ 0 & \text { if } \rho\left(a_{22}\right)>1\end{cases}
$$

where $\rho\left(a_{22}\right)$ is the spectral radius of the operator $a_{22}$ and $\beta\left(a_{33}\right)$ is the sum of multiplicities of eigenvalues of the operator $a_{33}$, greater than 1 .

\section{Index of a nonlinear operator}

Let $A$ be a nonlinear operator and let $Q$ be a closed convex set of a Banach space $X$ that is invariant under the operator $A$. In this part, we discuss the relative fixed point index calculations of $A$ under the assumption that $A$ has a Fréchet derivative at its fixed point. Recall that an operator $A$ is called differentiable at the point $x_{0}$ with respect to $Q$, if

$$
\lim _{\substack{x \in Q, x \rightarrow x_{0}}} \frac{\left\|A x-A x_{0}-A^{\prime}\left(x_{0}\right) x\right\|}{\|x\|}=0,
$$

and differentiable at infinity with respect to $Q$, if

$$
\lim _{\substack{x \in Q \\ x \rightarrow \infty}} \frac{\left\|A x-A^{\prime}(\infty) x\right\|}{\|x\|}=0
$$

where $A^{\prime}\left(x_{0}\right)$ and $A^{\prime}(\infty)$ are linear operators.

Let $Q$ be an arbitrary closed convex set of a Banach space $X$. Assume that $Q$ is invariant under a completely continuous operator $A$. Let $x_{0}$ be a fixed point of the operator $A$ and let $A$ have a Fréchet derivative at the point $x_{0}$ with respect to $Q$.

The following lemmas show that in the case when the fixed point $x_{0}$ is not the infinity singular point some wedge $W_{x_{0}}$ comprising the set $Q$ is invariant under the mapping of the derivative $A^{\prime}\left(x_{0}\right)$, whereas in the case of the infinity singular point some wedge $W_{\infty}$ lying in the set $Q$ is invariant under the mapping of derivative $A^{\prime}(\infty)$. 
Lemma 3.1. Let $A$ be a completely continuous operator, acting in a Banach space $X$, and let $Q \subseteq X$ be a closed convex set that is invariant under $A$. Let $x_{0} \in Q$ be a fixed point of the operator $A$ and let $A$ have Fréchet derivative at $x_{0}$ with respect to $Q$. Then the wedge

$$
W_{x_{0}}=\operatorname{cl}\left\{h=t\left(x-x_{0}\right): t \geq 0, x \in Q\right\}
$$

is invariant under the mapping of the derivative $A^{\prime}\left(x_{0}\right)$.

Proof. To prove that $W_{x_{0}}$ is actually a wedge it is sufficient to show that $W_{x_{0}}$ is closed under addition of its elements and their multiplication by nonnegative reals. If $h_{1}, h_{2} \in$ $W_{x_{0}}$, then $h_{1}=t_{1}\left(x_{1}-x_{0}\right), h_{2}=t_{2}\left(x_{2}-x_{0}\right)$, where $x_{1}, x_{2} \in Q$ and $0 \leq t_{1}, t_{2}<\infty$. Thus for any $\alpha$ and $\beta$ for which $0 \leq \alpha, \beta<\infty$, and $\alpha t_{1}+\beta t_{2}>0$, we have

$$
\begin{aligned}
\alpha h_{1}+\beta h_{2} & =\alpha t_{1}\left(x_{1}-x_{0}\right)+\beta t_{2}\left(x_{2}-x_{0}\right) \\
& =\left(\alpha t_{1}+\beta t_{2}\right)\left(\frac{\alpha t_{1}}{\alpha t_{1}+\beta t_{2}}\left(x_{1}-x_{0}\right)+\frac{\beta t_{2}}{\alpha t_{1}+\beta t_{2}}\left(x_{2}-x_{0}\right)\right) \\
& =\left(\alpha t_{1}+\beta t_{2}\right)\left(\frac{\alpha t_{1}}{\alpha t_{1}+\beta t_{2}} x_{1}+\frac{\beta t_{2}}{\alpha t_{1}+\beta t_{2}} x_{2}-x_{0}\right) .
\end{aligned}
$$

An element

$$
\frac{\alpha t_{1}}{\alpha t_{1}+\beta t_{2}} x_{1}+\frac{\beta t_{2}}{\alpha t_{1}+\beta t_{2}} x_{2}
$$

is a convex combination of elements $x_{1}, x_{2} \in Q$ and therefore it is in $Q$. Thus (3.4) implies $\alpha h_{1}+\beta h_{2} \in W_{x_{0}}$. If $\alpha t_{1}+\beta t_{2}=0$, then $\alpha h_{1}+\beta h_{2}=0$ and thus $\alpha h_{1}+\beta h_{2} \in W_{x_{0}}$.

Let $h$ be an arbitrary nonzero element of $W_{x_{0}}$. Then there exist $x \in Q$ and $t>0$ such that $h=t\left(x-x_{0}\right)$. From the differentiability of the operator $A$ at $x_{0}$ and the linearity of the operator $A^{\prime}\left(x_{0}\right)$ it follows that

$$
t\left(A x-x_{0}\right)=A^{\prime}\left(x_{0}\right) h+t \omega\left(\frac{h}{t}\right)
$$

Since $A(Q) \subseteq Q$, the element in the left-hand side of this equality is in $W_{x_{0}}$. Taking the limit as $t \rightarrow \infty$, by the closedness of $W_{x_{0}}$, we get $A^{\prime}\left(x_{0}\right) h \in W_{x_{0}}$. This completes the proof.

Lemma 3.2. Let $A$ be a completely continuous operator, acting in a Banach space $X$, and let $Q \subseteq X$ be a closed convex unbounded set that is invariant under A. Let $A$ have Fréchet derivative at infinity with respect to $Q$. Then the wedge

$$
W_{\infty}=\{h \in X: x+t h \in Q(x \in Q, 0 \leq t<\infty)\}
$$

is invariant under the mapping of the derivative $A^{\prime}(\infty)$.

Proof. To prove that $W_{\infty}$ is invariant under the operator $A^{\prime}(\infty)$, it suffices to show that there exists an element $x_{*} \in Q$ such that $x_{*}+t A^{\prime}(\infty) h \in Q$ for any $h \in W_{\infty}$ and $0 \leq t<\infty$. Let $x_{*} \in Q$, then for any $\lambda>0, t \geq 0, h \in W_{\infty}$ we have $x_{*}+\lambda t h \in Q$. Since $Q$ is invariant 
under the operator $A$, we get $A^{\prime}(\infty) x_{*}+\lambda t A^{\prime}(\infty) h+w\left(x_{*}+\lambda t h\right) \in Q$ for any $\lambda \geq 0, t \geq 0$, $h \in W_{\infty}$. Then, by the convexity of $Q$, for any $\lambda \geq 0$ we have

$$
\left(1-\frac{1}{\lambda}\right) x_{*}+\frac{1}{\lambda} A^{\prime}(\infty) x_{*}+t A^{\prime}(\infty) h+\frac{1}{\lambda} w\left(x_{*}+\lambda t h\right) \in Q
$$

Taking the limit as $\lambda \rightarrow \infty$, by the closedness of $Q$, we get $x_{*}+t A^{\prime}(\infty) h \in Q$ for any $t \geq 0$, $h \in W_{\infty}$. This completes the proof.

The following theorem specifies the main result of Dancer [2, Theorem 1].

Theorem 3.3. Let $A$ be a completely continuous operator, acting in a Banach space $X$, and let $Q \subseteq X$ be a closed convex set that is invariant under $A$. Let $x_{0} \in \partial Q$ be a fixed point of the operator $A$ and let $A$ have Fréchet derivative at $x_{0}$ with respect to $Q$. Then the wedge

$$
W_{x_{0}}=\operatorname{cl}\left\{h=t\left(x-x_{0}\right): t \geq 0, x \in Q\right\}
$$

is invariant under the mapping of the derivative $A^{\prime}\left(x_{0}\right)$.

If $A^{\prime}\left(x_{0}\right) x \neq x$ for $x \in W_{x_{0}}, x \neq 0$, then the fixed point $x_{0}$ of the vector field $\Phi=I-A$ is isolated in $Q$ and

$$
\text { ind }\left(x_{0}, I-A ; Q\right)=\text { ind }\left(0, I-A^{\prime}\left(x_{0}\right) ; W_{x_{0}}\right) \text {. }
$$

Proof. Without loss of generality it can be assumed that $x_{0}=0$ (in the opposite case, the whole argument needs to be made for the operator $\left.A\left(x_{0}+x\right)-x_{0}\right)$.

From the differentiability of the operator $A$ at the point 0 it follows that there exists a linear operator $B=A^{\prime}(0)$ such that

$$
A x=B x+w(x)
$$

where the operator $w$ meets the condition

$$
\lim _{\substack{x \in Q, x \rightarrow 0}} \frac{\|w(x)\|}{\|x\|}=0 .
$$

If $B x \neq x$ for $x \in W_{0}, x \neq 0$, there exists a positive real $c>0$ such that for any $x \in W_{0}$ the inequality

$$
\|x-B x\| \geq c\|x\|
$$

holds.

Choose a real $r>0$ such that inequalities

$$
\|A x-B x\| \leq \frac{c}{2}\|x\|, \quad \frac{c r}{(c+2\|B\|) \rho_{r}}-1>0
$$

hold for $x \in Q,\|x\| \leq r$, where $\rho_{r}=\sup _{x \in W_{0},\|x\|=r} \rho(x, Q)$ and $\rho(x, Q)$ denotes the distance from the point $x$ to the set $Q$. 
Show that on the intersection of the sphere $S_{r}=\{x \in X:\|x\|=r\}$ of radius $r$ with the set $W_{0}$ the vector field $\Phi^{\prime}=I-B$ is linearly homotopic to the field $\Phi_{\alpha} x=x-A P_{\alpha} x$ where $\alpha$ meets the condition

$$
0<\alpha<\frac{c r}{(c+2\|B\|) \rho_{r}}-1
$$

and $P_{\alpha}$ is a projection (in general, nonlinear) on $Q$ that has the following property:

$$
\left\|x-P_{\alpha} x\right\| \leq(1+\alpha) \rho(x, Q) \quad(x \in X)
$$

(the existence of such projection follows from [4, Theorem 18.1]).

To prove this, consider the linear deformation

$$
\Phi(\lambda, x)=x-(1-\lambda) B x-\lambda A P_{\alpha} x \quad\left(x \in S_{r} \cap W_{0}, \lambda \in[0,1]\right)
$$

that connects vector fields $\Phi^{\prime}$ and $\Phi_{\alpha}$. From the convexity of $W_{0}$ and the invariance of the sets $W_{0}$ and $Q \subseteq W_{0}$ under operators $B$ and $A$, respectively, it follows that the element $(1-\lambda) B x+\lambda A P_{\alpha} x$ is in $W_{0}$ for any $x \in S_{r} \cap W_{0}$ and $\lambda \in[0,1]$.

The nondegeneracy of the deformation $\Phi(\lambda, x)$ for $x \in S_{r} \cap W_{0}, \lambda \in[0,1]$ follows from the inequalities

$$
\begin{aligned}
\| x- & (1-\lambda) B x-\lambda A P_{\alpha} x \| \\
& \geq\|x-B x\|-\lambda\left\|A P_{\alpha} x-B x\right\| \\
& \geq\|x-B x\|-\left\|A P_{\alpha} x-B P_{\alpha} x\right\|-\left\|B x-B P_{\alpha} x\right\| \\
& \geq c r-\frac{c}{2}\left(r+(1+\alpha) \rho_{r}\right)-\|B\|(1+\alpha) \rho_{r}=\frac{c r}{2}-\left(\frac{c}{2}+\|B\|\right)(1+\alpha) \rho_{r}>0 .
\end{aligned}
$$

Hence the vector fields $\Phi^{\prime}$ and $\Phi_{\alpha}$ are homotopic on $S_{r} \cap W_{0}$. Thus, by the first property of the relative rotation,

$$
\text { ind }\left(0, I-B ; W_{0}\right)=\text { ind }\left(0, I-A P_{\alpha} ; W_{0}\right) \text {. }
$$

By definition, the relative index ind $\left(0, I-A P_{\alpha} ; W_{0}\right)$ is equal to the relative rotation $\gamma(I-$ $\left.A P_{\alpha}, B_{r} \cap W_{0} ; W_{0}\right)$ of the vector field $I-A P_{\alpha}$ on the boundary of an open set $B_{r} \cap W_{0}$, where $B_{r}=\{x \in X:\|x\|<r\}$. By the additivity property of rotation,

$$
\gamma\left(I-A P_{\alpha}, B_{r} \cap W_{0} ; W_{0}\right)=\gamma\left(I-A P_{\alpha}, B_{r} \cap Q ; W_{0}\right)+\gamma\left(I-A P_{\alpha}, B_{r} \cap\left(W_{0} \backslash Q\right) ; W_{0}\right) .
$$

From the fact that $A P_{\alpha}$ has no fixed points beyond $Q$, it follows that $\gamma\left(I-A P_{\alpha}, B_{r} \cap\right.$ $\left.\left(W_{0} \backslash Q\right) ; W_{0}\right)=0$. On the other hand, the relative rotation $\gamma\left(I-A P_{\alpha}, B_{r} \cap Q ; W_{0}\right)$ can be considered as the rotation $\gamma\left(I-A, B_{r} \cap Q ; Q\right)$ of the vector field $I-A$ on the boundary of the open set $B_{r} \cap Q$ with respect to $Q$. By the definition of relative index, this rotation coincides with ind $(0, I-A ; Q)$. This completes the proof.

As it appears, the analogous statement is true for the case of asymptotic index. 
Theorem 3.4. Let $A$ be a completely continuous operator, acting in a Banach space $X$, and let $Q \subseteq X$ be a closed convex unbounded set that is invariant under A. Let $A$ have Fréchet derivative at infinity with respect to $Q$. Then the wedge

$$
W_{\infty}=\{h \in X: x+t h \in Q(x \in Q, 0 \leq t<\infty)\}
$$

is invariant under the mapping of the derivative $A^{\prime}(\infty)$.

If $W_{\infty} \neq\{0\}$ and $A^{\prime}(\infty) x \neq x$ for $x \in W_{\infty}, x \neq 0$, then the infinity singular point of the vector field $\Phi=I-A$ is isolated in $Q$ and

$$
\operatorname{ind}(\infty, I-A ; Q)=\operatorname{ind}\left(0, I-A^{\prime}(\infty) ; W_{\infty}\right) .
$$

Proof. From the differentiability of the operator $A$ at infinity it follows that there exists linear operator $B=A^{\prime}(\infty)$ such that

$$
A x=B x+w(x)
$$

where the operator $w$ meets the condition

$$
\lim _{\substack{x \in Q \\ x \rightarrow \infty}} \frac{\|w(x)\|}{\|x\|}=0 .
$$

If $B x \neq x$ for $x \in W_{\infty}, x \neq 0$, then there exists a positive real $c>0$ such that for all $x \in W_{\infty}$ the inequality

$$
\|x-B x\| \geq c\|x\|
$$

holds.

Choose a real $R>0$ such that inequalities

$$
\|A x-B x\| \leq \frac{c}{2}\|x\|, \quad \frac{c R}{2(1+c+\|B\|) \rho_{R}}-1>0
$$

hold for all $x \in Q,\|x\| \geq R$, where $\rho_{R}=\sup _{x \in Q,\|x\|=R} \rho\left(x, W_{\infty}\right)$ and $\rho\left(x, W_{\infty}\right)$ denotes the distance from the point $x$ to the set $W_{\infty}$.

Show that on the intersection of the sphere $S_{R}=\{x \in X:\|x\|=R\}$ of radius $R$ with the set $Q$ the vector field $\Phi=I-A$ is linearly homotopic to the field $\Phi_{\alpha} x=x-B P_{\alpha}$, where a real $\alpha$ meets the condition

$$
0<\alpha<\frac{c R}{2(1+c+\|B\|) \rho_{R}}-1,
$$

and $P_{\alpha}$ is a projection (in general, nonlinear) on $W_{\infty}$ that has the following property:

$$
\left\|x-P_{\alpha} x\right\| \leq(1+\alpha) \rho\left(x, W_{\infty}\right) \quad(x \in X) .
$$

Consider the linear deformation

$$
\Phi(\lambda, x)=x-(1-\lambda) B P_{\alpha} x-\lambda A x \quad\left(x \in S_{R} \cap Q, \lambda \in[0,1]\right)
$$


that connects vector fields $\Phi$ and $\Phi_{\alpha}$. Since the convexity of $Q$ and the invariance of the sets $W_{\infty} \subseteq Q$ and $Q$ under the operators $B$ and $A$, respectively, it follows that the element $(1-\lambda) B P_{\alpha} x+\lambda A x$ is in $Q$ for any $x \in S_{R} \cap Q$ and $\lambda \in[0,1]$.

The nondegeneracy of $\Phi(\lambda, x)$ for $x \in S_{R} \cap Q, \lambda \in[0,1]$ follows from the inequalities

$$
\begin{aligned}
\| x- & (1-\lambda) B P_{\alpha} x-\lambda A x \| \\
& \geq\left\|x-B P_{\alpha} x\right\|-\lambda\left\|A x-B P_{\alpha} x\right\| \\
& \geq\left\|P_{\alpha} x-B P_{\alpha} x\right\|-\left\|x-P_{\alpha} x\right\|-\|A x-B x\|-\left\|B x-B P_{\alpha} x\right\| \\
& \geq c\left(R-(1+\alpha) \rho_{R}\right)-(1+\alpha) \rho_{R}-\frac{c R}{2}-\|B\|(1+\alpha) \rho_{R} \\
& =\frac{c R}{2}-(1+c+\|B\|)(1+\alpha) \rho_{R}>0 .
\end{aligned}
$$

Hence the vector fields $\Phi$ and $\Phi_{\alpha}$ are homotopic on $S_{R} \cap Q$. Thus, by the first property of the relative rotation,

$$
\operatorname{ind}(\infty, I-A ; Q)=\operatorname{ind}\left(\infty, I-B P_{\alpha} ; Q\right) \text {. }
$$

By definition, the relative index ind $\left(\infty, I-B P_{\alpha} ; Q\right)$ is equal to the relative rotation $\gamma(I-$ $\left.B P_{\alpha}, B_{R} \cap Q ; Q\right)$ of the vector field $I-B P_{\alpha}$ on the boundary of an open set $B_{R} \cap Q$, where $B_{R}=\{x \in X:\|x\|<R\}$. By the additivity property of rotation,

$$
\gamma\left(I-B P_{\alpha}, B_{R} \cap Q ; Q\right)=\gamma\left(I-B P_{\alpha}, B_{R} \cap W_{\infty} ; Q\right)+\gamma\left(I-B P_{\alpha}, B_{R} \cap\left(Q \backslash W_{\infty}\right) ; Q\right) .
$$

From the fact that the operator $B P_{\alpha}$ has no fixed points beyond $W_{\infty}$, it follows that the relative rotation $\gamma\left(I-B P_{\alpha}, B_{R} \cap\left(Q \backslash W_{\infty}\right) ; Q\right)$ is equal to zero. Finally, the relative rotation $\gamma\left(I-B P_{\alpha}, B_{R} \cap W_{\infty} ; Q\right)$ can be considered as the rotation $\gamma\left(I-B, B_{R} \cap W_{\infty} ; W_{\infty}\right)$ of the vector field $I-B$ on the boundary of open set $B_{R} \cap W_{\infty}$ with respect to $W_{\infty}$. By the definition of relative index and since the operator $B$ has no nonzero fixed points in $W_{\infty}$, the latter rotation coincides with ind $\left(0, I-B ; W_{\infty}\right)$. This completes the proof.

\section{References}

[1] E. N. Dancer, On the indices of fixed points of mappings in cones and applications, Journal of Mathematical Analysis and Applications 91 (1983), no. 1, 131-151.

[2] __ Positivity of maps and applications, Topological Nonlinear Analysis: Degree, Singularity, and Variations, Progr. Nonlinear Differential Equations Appl., vol. 15, Birkhäuser Boston, Massachusetts, 1995, pp. 303-340.

[3] Y. Y. Isaenko, The fixed point index of linear positive operators, Trudy Aspirantov Voronezhskogo Universiteta (Proceedings of Postgraduate Students of Voronezh State University) (1969), 54-58 (Russian).

[4] M. A. Krasnosel'skiǔ and P. P. Zabreiko, Geometrical Methods of Nonlinear Analysis, Nauka, Moscow, 1975, translated into English: Springer, Berlin, 1984.

[5] E. M. Mukhamadiev and T. Sabirov, On the computation of the relative index of the zero of a vector field, Trudy Seminara po Funktional'nomu Analizn (Proceedings of Seminar on Functional Analysis) 12 (1969), 116-121 (Russian). 
[6] Y. V. Pokornyi, Relative indices of positive operators, Trudy Matematicheskogo Fakul'teta Voronezhskogo Universiteta (Proceedings of Department of Mathematics of Voronezh State University) 4 (1970), 79-89 (Russian).

[7] Y. V. Pokornyi and I. N. Astaf'eva, On the index at zero and at infinity for positive nonlinear operators, Uspehi Natematicheskih Nauk 26 (1971), no. 3, 189 (Russian).

[8] P. P. Zabrejko, Rotation of vector fields: definition, basic properties, and calculation, Topological Nonlinear Analysis, II: Degree, Singularity, and Variations (Frascati, 1995), Progr. Nonlinear Differential Equations Appl., vol. 27, Birkhäuser Boston, Massachusetts, 1997, pp. 445-601.

A. V. Guminskaya: Mechanics and Mathematics Faculty, Belarusian State University, Independence Avenue 4, 220050 Minsk, Belarus

E-mail address: aguminskaya@yandex.ru

P. P. Zabreiko: Mechanics and Mathematics Faculty, Belarusian State University, Independence Avenue 4, 220050 Minsk, Belarus

E-mail address: zabreiko@mail.ru 


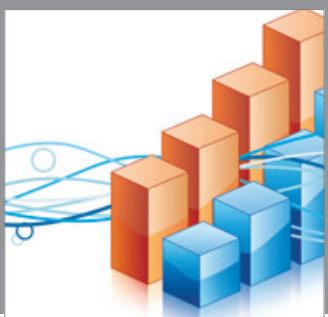

Advances in

Operations Research

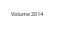

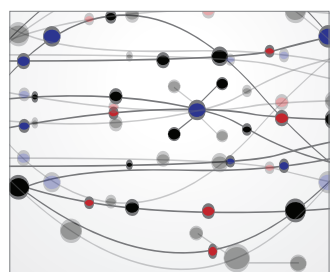

\section{The Scientific} World Journal
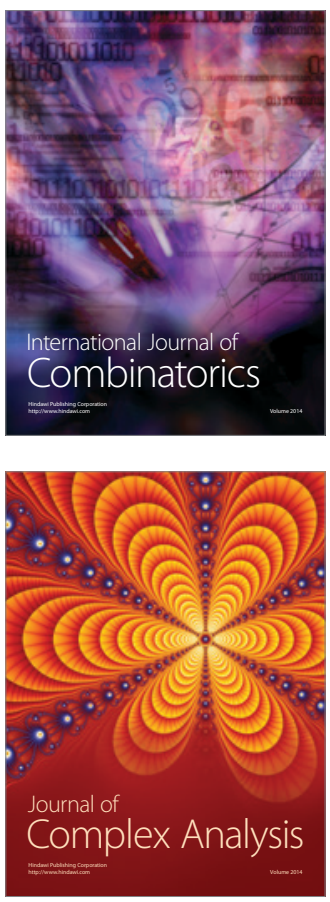

International Journal of

Mathematics and

Mathematical

Sciences
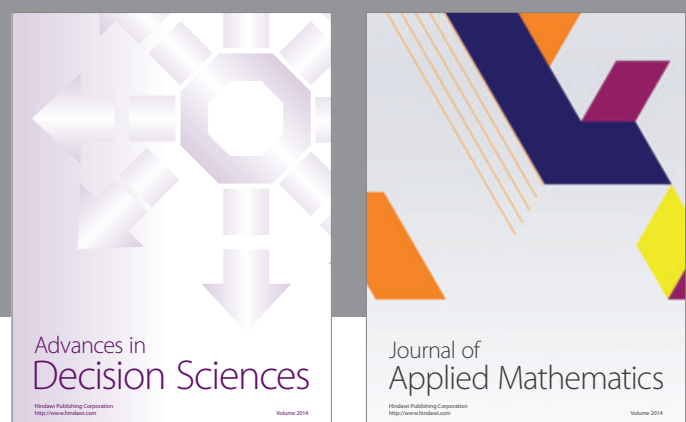

Journal of

Applied Mathematics
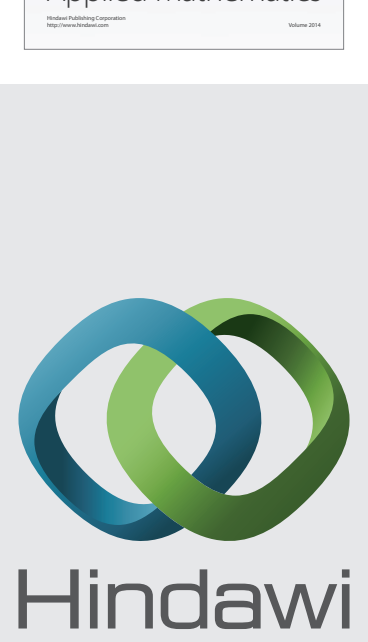

Submit your manuscripts at http://www.hindawi.com
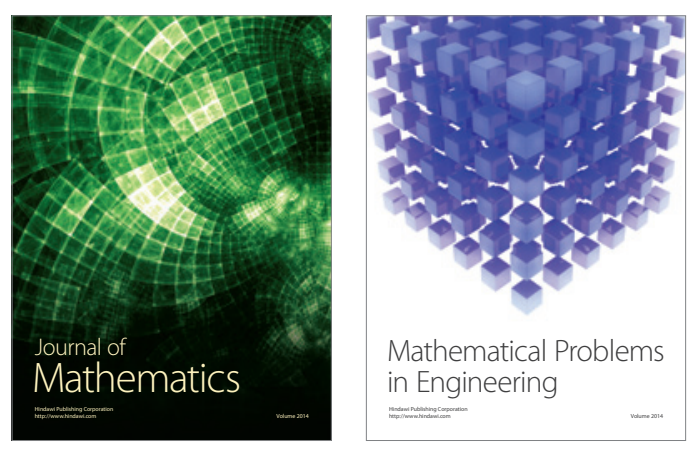

Mathematical Problems in Engineering
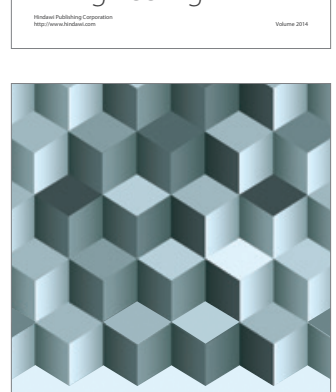

Journal of

Function Spaces
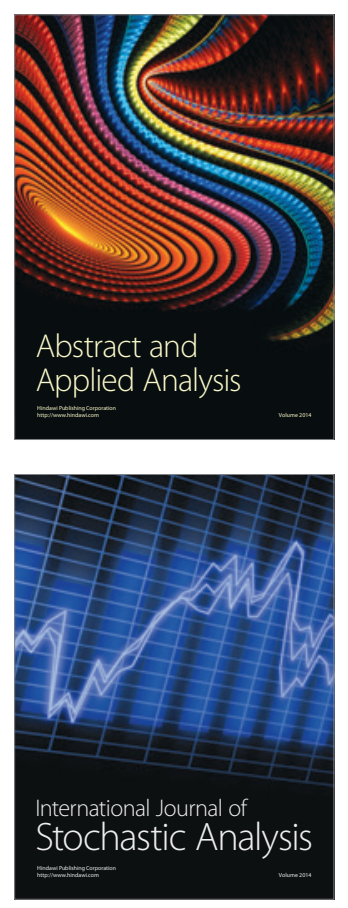

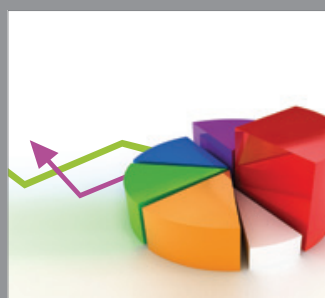

ournal of

Probability and Statistics

Promensencen
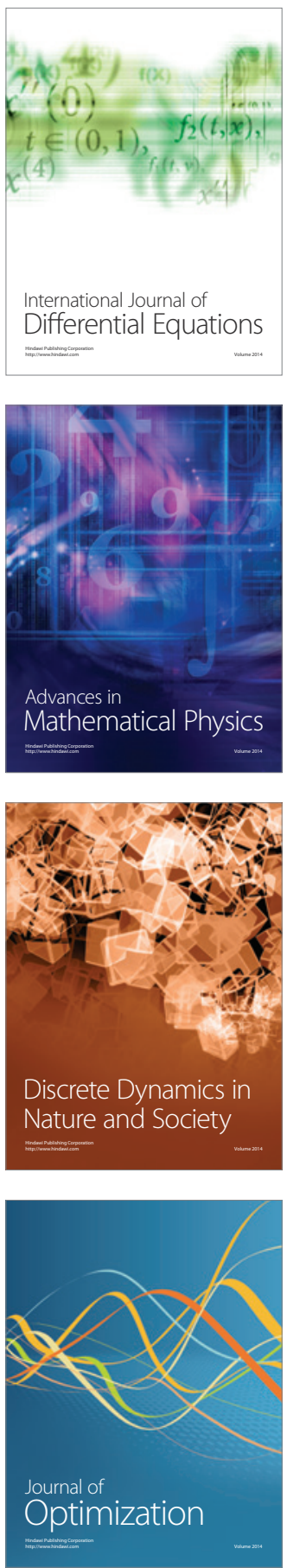